\section{Los temas de salud en la Unión Europea: su impacto en la sanidad española}

\author{
Health policy in the European Union: impact \\ on the Spanish health system
}

Luisa Guimarães 1

José-Manuel Freire 2

\footnotetext{
${ }^{1}$ Escola Nacional de Saúde Pública Sergio Arouca, Fundação Oswaldo Cruz, Rio de Janeiro, Brasil. 2 Escuela Nacional de Sanidad, Instituto de Salud Carlos III, Madrid, España.

Correspondencia L. Guimarães Escola Nacional de Saúde Pública Sergio Arouca, Fundação Oswaldo Cruz. Rua Leopoldo Bulhões 1480, Rio de Janeiro, $R J$ 21041-210, Brasil. luisa.guimaraes@solar.com.br
}

\begin{abstract}
This text reviews the impact of European integration on the health sector (public health and health services) by studying European Union $(E U)$ institutions, functioning, and responsibilities through the literature, documents, and authors' observations. The EU does not have direct health responsibilities, but Community legislation has important repercussions on all member states' health policies. This influence affects health protection issues, consumer safety, regulation of medicines and medical devices, mutual recognition of professional qualifications, freedom of movement for health professionals and patients, public contracts and bidding, research, etc. The evolution of EU health policy shows a progressive reinforcement of responsibilities consistent with the objective of reaching a high level of health protection, which in turn affects other European policies. The impact of European integration on the Spanish health system is analyzed as a case study, and key aspects and present and future challenges are highlighted. Lessons are also drawn for regional integration processes to foster equity and efficiency in health.
\end{abstract}

European Union; Health Policy; Health Systems

\section{Introducción}

La Unión Europea (UE) es el resultado, único en la historia, de la integración pacífica de Estados soberanos a través de tratados que transfieren competencias a una instancia supranacional, sin sustituir a los gobiernos nacionales 1. La construcción de la UE aporta un mensaje de paz y seguridad, de desarrollo económico con solidaridad y cohesión social, resumido en lo que se conoce como modelo social europeo.

En la UE la sanidad, en su doble dimensión - salud pública y asistencia sanitaria -, es competencia exclusiva de los Estados Miembros, y sin embargo, la legislación comunitaria ha tenido en ella un importante impacto. Por ello, su estudio es de gran interés para conocer e influir en su dinámica y aprovechar las lecciones que se puedan aplicar en otros procesos de integración, como MERCOSUR.

Para comprender el impacto de la Unión en el sector salud es imprescindible una visión de conjunto de las instituciones, actores, competencias y mecanismos de funcionamiento, y del principio europeo de subsidiariedad, según el cual la intervención de la UE en ámbitos que no correspondan a su competencia se justifica cuando los Estados Miembros no puedan alcanzar los objetivos previstos o cuando la acción se pueda cumplir mejor en el ámbito de la Unión.

La influencia de las directivas de la Unión en sanidad se manifiesta a través de tres grandes 
vías. La primera y más importante es el desarrollo del mercado interior que ampara y regula la libertad de movimiento dentro de la UE de personas, bienes, servicios y capital. El mercado interior ha tenido gran impacto en los estándares de protección de la salud y seguridad de los consumidores europeos, así como en sus servicios sanitarios a través de la regulación de medicamentos, aparatos médicos, contratación publica, homologación de títulos profesionales y, en menor medida, en la libertad de movimiento de pacientes y profesionales. La segunda vía es el mandato para proteger la salud pública y a los consumidores que hace del alto nivel de protección de la salud un objetivo comunitario. La tercera vía es la investigación biomédica que está recibiendo significativo impulso en la actualidad, pero que por su especificidad no será analizada aquí.

En este trabajo se ofrece una visión general del impacto de la Unión en el sector salud a partir de revisiones bibliográficas, análisis de documentos, visitas a instituciones, observaciones coyunturales y experiencia de uno de los autores en la administración sanitaria española y en la UE. Se realizaron entrevistas semi-estructuradas a responsables de temas europeos en las administraciones sanitarias central y regional españolas y así como a académicos. El tema de interés ha sido analizar el proceso de integración europea, el funcionamiento de la UE y sus repercusiones el sector sanitario, con la hipótesis de que el mercado interior europeo condiciona diversos aspectos de los sistemas sanitarios. El texto aborda en primer lugar el funcionamiento de UE a partir de los tratados e instituciones; a continuación revisa los temas de salud en las políticas europeas, y el impacto en los sistemas sanitarios europeos de la libre circulación de bienes, servicios, personas y capitales. Posteriormente, como ilustración, se estudia el impacto de la integración europea en la sanidad española. Finalmente, se destacan algunas lecciones para protección de la salud y desarrollo equitativo y eficiente de los servicios sanitarios en procesos de integración transnacionales.

Durante los últimos años la Unión ha reforzado sus responsabilidades en salud pública tanto en los tratados como en la Constitución Europea, en proceso de ratificación. Este refuerzo se manifiesta en importantes iniciativas como la Estrategia Sanitaria Europea, el sistema de alerta ante las amenazas sanitarias, la creación de varias agencias relacionadas con la salud. Muchos Estados Miembros están abordando reformas sectoriales en salud y ésta, en un sentido amplio, es objeto de un amplio abanico de políticas públicas relacionadas con iniciativas comunitarias, en áreas como medio ambiente, agricultura y alimentación, recursos humanos. Para España, con un sistema político y sanitario totalmente descentralizado en sus 17 Comunidades Autónomas, la Unión impone al Gobierno central el doble desafío de negociar primero en las instituciones de la UE y que luego trasladar las políticas a las Comunidades Autónomas para su implementación.

Este texto ha sido parte del Programa de Doctorado en Salud Pública, línea de investigación Políticas y Sistemas de Salud en Perspectiva Comparada, de la Escuela Nacional de Salud Pública Sergio Arouca, Fundación Oswaldo Cruz, Ministerio de Salud de Brasil.

\section{La Unión Europea}

Desde el 1 de enero de 2007 la UE incluye veinticinco países, 460 millones de habitantes y cuatro millones de kilómetros cuadrados de superficie 2 . Es el mercado más grande del mundo con libertad de circulación de personas, bienes, servicios y capitales. El euro es una manifestación palpable de los logros de la Unión para la vida de los europeos y la economía mundial. Todavía "Europa no es una patria consolidada y no se reacciona ante ella de la misma manera que ante la propia identidad" 3 (p. 7); la ciudadanía europea no reemplaza la ciudadanía nacional, sino que la complementa; la Unión es, “desde sus inicios, un proyecto con motivaciones y objetivos políticos que se ha ido concretando por la vía económica" 3 (p. 7). Los Estados Miembros siguen siendo naciones soberanas que comparten competencias, delegan poderes específicos a las instituciones comunitarias para actuar en el interés común. La UE es el resultado de sucesivos tratados, firmados a partir de 1951, de la incorporación gradual de nuevos miembros 1,4, y del desarrollo institucional 5 para cumplir los compromisos acordados y representar a los Estados Miembros y a sus ciudadanos 6, asimismo su construcción se hace al caminar 7 .

Las instituciones europeas deben actuar coordinadamente en un entorno en el que, si bien se comparten objetivos comunes, existen multitud de intereses en conflicto, que demandan procedimientos sofisticados y detallados de conciliación ${ }^{8}$. La toma de decisiones requiere la intervención de diferentes instancias y de una pluralidad de comités, grupos de trabajo y foros 3 . El balance entre lo nacional y lo comunitario es fundamental y se delimita por competencias y principios que equilibran el papel de las instituciones comunitarias. Las competencias de la Unión son clasificadas en exclusivas (política económica), compartidas (mercado 
interior) y de apoyo (coordinación y apoyo a las acciones de los miembros) ${ }^{9}$. El principio de subsidiariedad impone que la Unión sólo interviene cuando la acción comunitaria es más eficaz que la nacional y, el principio de proporcionalidad establece que las competencias, el contenido y la forma de la acción de la UE se basan en los objetivos del Tratado 4,6,10,11.

\section{Unión Europea y el sector salud}

La complejidad de la arquitectura y funcionamiento comunitario tiene en la salud un observatorio especialmente interesante. Las directivas europeas repercuten en la sanidad de forma compleja y distinta para cada uno de los Estados Miembros 12,13; de una forma directa y obligatoria en los temas de protección de la salud, y más indirectamente, pero con un gran impacto, a través de las políticas derivadas del mercado interior.

Aunque la Unión tiene un notable impacto en las políticas sanitarias de los Estados Miembros, los servicios de salud son competencia exclusiva de los Estados Miembros, y en modo alguno se contempla la armonización comunitaria de los sistemas o seguros de salud, tanto por la diversidad y complejidad sectorial de la sanidad en los Estados Miembros, como por el temor a que signifique más regulación o pérdida de estándares alcanzados 14 .

\section{Los temas de salud en los tratados}

Los tratados, centrados en aspectos económicos y políticos, incluyen sin embargo temas generales de protección de la salud. La salud pública como competencia comunitaria aparece por primera vez en 1992, en el Tratado de Maastricht 15 (Art. 129-129A). En 1997, el Tratado de Ámsterdam 16 (Art. 3 y 152) refuerza la implicación comunitaria en salud pública, estableciendo que debe asegurarse un alto nivel de protección a la salud en la definición e implementación de todas las políticas y actividades que puedan influir en la salud. En 2000, la Estrategia de Lisboa refleja la decisión del Consejo Europeo de renovar la meta europea del crecimiento económico sostenible con cohesión social, siendo la salud objeto de interés específico por considerarse que tiene impacto positivo en el crecimiento económico 17,18.

Dada la responsabilidad exclusiva de los Estados en la organización y financiación sanitaria, para garantizar la protección a la salud la Unión interviene sólo a través de los mecanismos del mercado interior, en aquellos temas de salud pública que traspasan las fronteras de los Estados 15 .
La Comisión Europea, bajo el principio de subsidiariedad, legisla y vigila el cumplimiento por los Estados Miembros de las Directivas comunitarias para garantizar tanto la protección de la salud en el mercado interior, como para hacer efectiva la libertad de circulación.

Los temas de salud en las instituciones europeas

Dentro de la Comisión Europea no existe un equivalente a los Ministerios de Salud de los Estados Miembros y los temas de salud son abordados por varias Direcciones Generales, sin embargo existe una Dirección General de Salud Pública y Protección de los Consumidores (DG Sanco) que focaliza los temas de salud dentro de la Comisión. Está organizada en tres áreas: (i) seguridad alimentaria (producción, procesamiento y venta de alimentos, salud animal y fitosanidad); (ii) protección de los consumidores (riesgos sanitarios, seguridad de productos y servicios, promoción de la salud y seguridad en las políticas europeas), y (iii) Salud Pública (indicadores sanitarios, determinantes de salud, comités científicos y enfermedades) 19 .

Para garantizar el alto nivel de protección de la salud que exigen los tratados, la DG Sanco ha integrado las acciones de protección y promoción de la salud en la Estrategia Sanitaria Europea, movilizando y coordinado las actividades de los gobiernos europeos, instituciones y organizaciones profesionales apoyando, por ejemplo, iniciativas e investigaciones sobre movilidad de pacientes, atención médica transfronteriza, movimiento de profesionales, centros de referencia, evaluación de tecnologías, información, sistemas sanitarios, seguridad de los pacientes 20 . Además, se han creado en la UE siete agencias relacionadas con temas de salud 6 . Por su parte, la participación social cuenta con en el Foro de Política de Salud de la Unión Europea, un organismo consultivo, vinculado a DG Sanco, integrado por representantes no gubernamentales y empresariales.

\section{Salud pública: protección y promoción de la salud en la Unión Europea}

Los programas y actividades desplegados por la DG Sanco dotan de visibilidad las políticas comunitarias de protección y promoción de la salud 19,20,21, amplían el campo tradicional 22 de la Salud Pública al incluir aspectos de cohesión social y medio ambiente, y el objetivo de reducir las desigualdades.

El desarrollo del mercado interior hace necesario que la Unión impulse y coordine activa- 
mente políticas de protección de la salud en un espacio en el que no existen ya fronteras para personas, bienes y servicios. Ello obliga a garantizar el mismo alto nivel de protección a la salud en todos los Estados a través de políticas de prevención de riesgos y promoción de condiciones y determinantes positivos para la salud, que son, a la vez, consecuencia y exigencias del propio mercado interior y de unas reglas de competencia que obligan a estándares comunes. Se exige un mismo alto nivel, común a toda la Unión, de seguridad e higiene en el trabajo para la protección de la salud de los trabajadores, de protección del medio ambiente, de modo que la competencia esté basada en innovación, productividad y calidad, pero no en bajos costes por deficiente seguridad en el trabajo, contaminación ambiental o riesgo para los consumidores. Los productos alimenticios constituyen un ejemplo obvio y especialmente importante de la necesidad de garantizar estándares comunes de protección de la salud de los consumidores en productos que se pueden mover libremente por toda la UE. De este modo, la protección de la salud se ha ido desarrollando en paralelo a la construcción del mercado interior común y sus reglas de competencia 14 .

\section{Sistemas de salud de los Estados Miembros y mercado interior}

Los Estados Miembros comparten objetivos de universalidad y de solidaridad en sus sistemas de protección social, a pesar de los distintos formatos políticos, financiación y organización de sus servicios. La Unión exige a los Estados Miembros cumplir las reglas del mercado interior, y con las restricciones macroeconómicas impuestas por los límites al déficit público, lo cual implica conciliar objetivos, en ocasiones contradictorios, económicos y sociales 23,24. Si bien los Estados Miembros tienen todas las competencias sobre sus servicios sanitarios, el mercado interior exige que el acceso a los servicios sanitarios no sea un obstáculo a la movilidad de ciudadanos, trabajadores y profesionales dentro de la UE 25.

El impacto del proceso de integración europea en los sistemas sanitarios de los Estados Miembros varía según sus sistemas de financiación y organización, pero es una realidad que afecta a todos países y sistemas 12,21,26. El mercado interior ha tenido importantes repercusiones tanto en la salud pública, como en los servicios sanitarios 1,12,13,14,21. Estas repercusiones se manifiestan, entre otros aspectos, a través de la obligada transposición de la normativa europea, en los derechos individuales de los consumidores en relación con la protección de la salud públi- ca, en los estándares comunes de seguridad alimentaria y de medio ambiente, en realidad de la movilidad de profesionales sanitarios, en la regulación de equipos médicos y medicamentos, en reglas para la contratación pública, y en garantías de prestación sanitaria a turistas y trabajadores comunitarios.

Mediosiglodetransformacioneseintegración en Europa muestran que el bienestar derivado del modelo europeo de protección social 23,24,27 posee poderosos lazos estabilizadores. En sanidad, ajustándose al principio de subsidiariedad, la Unión ha promovido estándares comunes, incentivando la protección a la salud y la conciliación de los objetivos de los sistemas sanitarios nacionales con el modelo social europeo y el mercado común interno. Aunque de forma no homogénea ${ }^{28}$, los servicios de salud han logrado en la UE su universalidad, un alto nivel de equidad en el acceso y el aumento progresivo del peso de los impuestos generales en su financiación 23

\section{Los sistemas sanitarios} en la Unión Europea

Europa es la cuna del estado de bienestar, uno de cuyos pilares son los servicios sanitarios que presentan gran diversidad entre sí $24,27,29$, aunque con patrones comunes de financiación pública y cobertura universal. En la UE se pueden distinguir dos grandes grupos de sistemas sanitarios: los sistemas de seguridad social y los sistemas tipo Servicio Nacional de Salud, atendiendo al origen de su financiación 24,30. Por sus características, los sistemas tipo Servicio Nacional de Salud, por ser públicos integrados, son los que presentan mayores problemas para la libre circulación de pacientes dentro de la UE; por el contrario, los sistemas de seguridad social, en los que el asegurador-financiador está diferenciado de los proveedores, se prestan más al cruce de fronteras nacionales para recibir atención médica.

A pesar de la solidez del modelo social europeo, la dinámica de la economía de mercado presiona continuamente para la revisión de las reglas de juego, en un delicado equilibrio entre protección social y crecimiento económico, como refleja la Estrategia de Lisboa 14,17. En esta arena político-económica, las tensiones para que los Estados mantengan servicios universales, solidarios y políticas públicas saludables pueden llevar a ceder terreno a las exigencias de una mayor competitividad de la economía europea 14, un dilema muy actual 18,22. 
El libre movimiento de bienes, servicios y capital en salud en el mercado interior

Los bienes, servicios y capital relacionados con la salud representan un sector que moviliza el $15 \%$ de las actividades económicas europeas, un monto significativo de recursos 26 . Los servicios médicos han sido retirados por el Parlamento Europeo de la Directiva sobre libre circulación servicios en la UE, reconociendo su carácter nacional y su difícil encaje en el mercado.

No obstante, muchas otras directivas afectan plenamente al sector salud, entre ellas, la Directiva sobre contratación pública que obliga a publicitar, a nivel europeo, compras y obras por encima de una cierta cuantía. Ello contribuye a la transparencia, objetividad, a ampliar el mercado y la competitividad. A pesar de ello, también aumenta la burocracia y el tiempo de los procesos, favoreciendo a las grandes corporaciones y exigiendo importantes adaptaciones sectoriales 12,21.

La UE tiene un papel determinante en dos importantes bienes sanitarios: aparatos médicos y medicinas. Los aparatos médicos constituyen un sector de gran importancia económica, tecnológica y sanitaria que abarca cerca de ocho mil productos. La regulación europea, muy apoyada por la industria, pretende garantizar calidad y seguridad, establecer estándares comunes que amplíen el mercado y favorezcan la libertad de circulación y la competencia.

Los medicamentos son uno de los sectores más regulados por la UE, que legisla desde la protección de patentes a las condiciones para la aprobación, distribución y publicidad de las medicinas, aunque los precios, sin embargo, son responsabilidad de los Estados Miembros. Para hacer operativa la política de la UE sobre productos farmacéuticos, se creó la Agencia Europea de Medicamentos (EMEA), responsable de la protección y promoción de la salud pública y animal, mediante evaluación y supervisión de los medicamentos de uso humano y veterinario ${ }^{31}$. La EMEA coordina las agencias de medicamentos de los Estados Miembros, facilita la comunicación sobre reacciones adversas, ensayos clínicos y el acceso compartido a bases de datos de medicamentos. Además, gestiona la aprobación centralizada de medicamentos, generando, a partir de una única solicitud, una autorización válida para toda la unión, correspondiendo luego a cada Estado autorizar su comercialización y precio en su territorio.

\section{El libre movimiento de personas en el} mercado interior: trabajador, paciente, turista y profesionales sanitarios

Los reglamentos comunitarios buscan, al favorecer la libre circulación de personas, garantizar los derechos sociales adquiridos, eliminar barreras territoriales y regular la competencia entre Estados. En consecuencia, el trabajador que se desplaza tiene sus derechos de seguridad social - períodos y cuantías de cotización - asegurados. Las pensiones contributivas, por accidente de trabajo y enfermedad profesional y los subsidios por defunción se podrán percibir en cualquier Estado Miembro, abonando el Estado de residencia las prestaciones directamente al beneficiario. Para ello, existen acuerdos europeos de intercambio electrónico de datos de seguridad social y procedimientos administrativos de adquisición, concesión y pago de derechos a los trabajadores 32 .

El acceso a los servicios sanitarios es garantizado por formularios específicos y por la Tarjeta Sanitaria Europea que acredita la portabilidad de derechos en salud durante los desplazamientos por Europa en estancias temporales en igualdad de condiciones con los nacionales 1 . Sin embargo, la Tarjeta Sanitaria Europea no es válida si el motivo del desplazamiento es recibir tratamiento médico; en este caso se necesita una autorización específica previa del Estado donde el ciudadano está asegurado 1,32,33.

La búsqueda de servicios sanitarios en otro Estado ha sido objeto de varias sentencias del Tribunal de Justicia Europeo ${ }^{1}$ que favorecen, con ciertas condiciones, el derecho de los pacientes a buscar atención médica en otros Estados Miembros, y reconocen que los Estados son libres para organizar la planificación, organización y financiamiento de sus servicios sanitarios. La reglas de acceso, en cuanto barreras a la libertad de circulación de bienes y servicios de salud se justifican para proteger la sostenibilidad y equidad de los sistemas sanitarios nacionales. No obstante, el Tribunal ha sentenciado que no se puede negar permiso para el tratamiento en otro Estado si existe indicación médica según criterios internacionales, aunque no sea normal en el país de origen, y tampoco si en el país de origen el tratamiento es proporcionado con retraso indebido 32,33,34. Las incertidumbres que esta situación provoca han llevado a la comisión a publicar a finales de 2006 un documento de consulta para clarificar la normativa existente sobre atención médica transfronteriza y explorar vías de cooperación entre los sistemas sanitarios de los Estados Miembros 35.

Por último, el mercado interior, con el reconocimiento mutuo de títulos académicos, ha su- 
primido de hecho las fronteras internas para los profesionales europeos, incluidas todas las profesiones sanitarias (medicina y sus especialidades, enfermería, odontología, farmacia, y otras). A pesar de que ello, y aunque persisten desequilibrios en los Estados Miembros entre oferta-demanda de distintas profesiones sanitarias, así como en rentas y condiciones de trabajo, el movimiento de profesionales es todavía marginal 36 , pero es un factor que tendrá una importancia creciente para el mercado de trabajo sanitario en toda la UE.

\section{Impacto de la Unión Europea en la sanidad española}

España ingresó en la UE en 1986. Desde entonces ha experimentado un salto cualitativo con grandes transformaciones y avances en su economía, infraestructuras y en el desarrollo de sus regiones más atrasadas, siendo en la actualidad la octava economía mundial. La pertenencia a la UE ha sido un factor de gran importancia para este progreso, tanto por los intangibles de confianza y estabilidad de todo tipo aportados por la pertenencia a la Unión, como por haber sido uno de los Estados que más Fondos de Cohesión ha recibido de la UE 8.

Tras la Constitución de 197837 España adopta la forma política de monarquía parlamentaria, con un Estado formado por diecisiete Comunidades Autónomas, que en la práctica hacen de España un país federal. Aunque los gobiernos regionales europeos no participan directamente en las instituciones de la UE 38,39 , de la cual son miembros exclusivamente los Estados, existe una presión creciente y un ambiente favorable a incorporar la visión e intereses de las regiones europeas, a la conformación de las voluntades nacionales dentro de la UE.

En el mismo de año de ingreso en la UE, 1986, durante la primera legislatura socialista, se aprobó la Ley General de Sanidad (Ley 14/1986) 40. Ésta creó el Sistema Nacional de Salud 41, tomando como base la Asistencia Sanitaria de la Seguridad Social 42, consolidando en España un modelo sanitario tipo Servicio Nacional de Salud. El Sistema Nacional de Salud, definido en la Ley General de Sanidad como el conjunto de los servicios de salud de las Comunidades Autónomas y del Estado, se caracteriza por su universalidad de facto, atención integral a la población, mayoritariamente con medios públicos, financiación por impuestos y gestión descentralizada en las diecisiete Comunidades Autónomas.

El Gobierno central y las Comunidades Autónomas comparten responsabilidades en los te- mas de salud: el Gobierno central tiene competencias exclusivas sobre las bases y coordinación general del Sistema Nacional de Salud, sanidad exterior, relaciones y acuerdos internacionales y legislación, medicamentos, formación sanitaria especializada, sistema de información y alta inspección. Las Comunidades Autónomas tienen competencias en Salud Pública, planificación sanitaria y asistencia sanitaria, al haber asumido todas ellas la gestión de la Asistencia Sanitaria de la Seguridad Social. En España los municipios no tienen responsabilidad alguna en la gestión de los servicios sanitarios públicos, sin embargo, tienen papel importante de salud pública (saneamiento, medio ambiente, higiene y seguridad alimentaria) 43,44 .

La transposición de reglamentos europeos al ordenamiento interno fue particularmente intensa entre 1992-1994, referente a la libre circulación de bienes sanitarios (medicamentos y aparatos médicos) y no sanitarios (fundamentalmente alimentos) y de personas (profesionales y ciudadanos comunitarios). Es importante resaltar que el mayor impacto sanitario de la integración de España en la UE ha tenido lugar en los temas directa o indirectamente relacionados con la protección de la salud y la seguridad de los consumidores. Es en los temas de salud pública donde la UE ha tenido un mayor desarrollo normativo y es la coordinación de estas políticas la que ha requerido un mayor esfuerzo por parte del Ministerio de Sanidad y Consumo, a través de la Dirección General de Salud Pública, y por parte del Ministerio de Agricultura, co-responsable en temas de seguridad e higiene alimentaria y de sanidad veterinaria, cuya importancia mostró la crisis de las vacas locas.

La normativa europea ha modificado la formación de médicos, dentistas, enfermeros y otros profesionales; así, a partir del 1 de enero de 1995, en cumplimiento de la Directiva 86/457/CEE, es obligatoria la formación reglada de postgrado (residencia) para ejercer la medicina en atención primaria. Igualmente, ha habido que adaptar las directivas europeas aspectos como el tiempo máximo de trabajo, una medida con importantes repercusiones en la organización de la atención continuada de los servicios sanitarios.

El libre movimiento de profesionales sanitarios en la UE está teniendo también cierto impacto en el Sistema Nacional de Salud; numéricamente todavía no es muy importante, pero apunta un notable potencial de incremento. Dados los diferenciales de renta y condiciones de trabajo existentes entre España y otros Estados Miembros, son los profesionales sanitarios españoles (médicos, enfermeras) los que tienden a emigrar a otros países de la UE, como Reino Uni- 
do, Portugal, Suecia. Al mismo tiempo empieza a hacerse evidente la escasez de profesionales sanitarios en ciertas especialidades y partes de España que, de momento, es cubierta con la importación de profesionales de América Latina y de los nuevos países del Este de la UE.

La contratación pública de bienes y servicios en el sistema sanitario debió igualmente adaptarse a las normas comunitarias de transparencia y competencia. Ello requirió nueva legislación específica nacional, con importante impacto en un área compleja en la que frecuentemente los concursos deben ser de ámbito comunitario europeo por la cuantía de los recursos implicados en sus compras u obras.

Una gran parte de las reformas habidas en política de medicamentos deriva de la pertenencia la Unión: actualización de los procedimientos de autorización y reconocimiento del derecho de patente de producto, y la creación de la Agencia Española de Medicamentos y Productos Sanitarios en 1997. Es importante señalar que en España, hasta el 1986, no se requería demostración de la eficacia basada en ensayos clínicos para autorizar un medicamento, sino sólo su seguridad y calidad 45; no existía patente de producto - la que prohíbe su fabricación y comercialización a los laboratorios que tengan la patente -, sino sólo de procedimiento - se prohibía la fabricación y comercialización de un producto por un procedimiento ya patentado, pero no por cualquier otro proceso que implicara una mínima variación del procedimiento. La patente de producto quedó protegida a partir de 1992, por legislación nacional de 1986, con una duración de veinte años a partir de la fecha de solicitud, más cinco años adicionales con el establecimiento del certificado complementario de protección de la patente 31 . No obstante, la fijación de precios y la financiación de medicamentos siguen siendo responsabilidades del Ministerio de Sanidad y Consumo 31,46. El alto peso económico de los medicamentos en el Sistema Nacional de Salud español es un buen indicador del gran impacto que la regulación europea ha tenido en la sanidad del país.

El ingreso en la Unión ha tenido también impacto en la demanda por servicios sanitarios por parte de ciudadanos comunitarios 21,32, obligando a regular la cobertura sanitaria de pacientes comunitarios dentro de España 13,21. España es uno de los principales destinos turísticos mundiales - más de 40 millones/año, la mayoría europeos -; su clima atrae a los pensionistas de otros Estados miembros, e incluso es uno de los destinos preferidos del Programa Erasmus de intercambio universitarios. La percepción positiva que existe sobre el Sistema Nacional de Salud es- pañol, que ofrece seguridad ante la enfermedad y accidentes, es sin duda uno de los factores que contribuye a este atractivo de España 13.

En cumplimiento al derecho europeo, los comunitarios acceden al Sistema Nacional de Salud en igualdad de condiciones con los nacionales. Los hospitales y centros de salud remiten información sobre la atención prestada a ciudadanos comunitarios a los servicios de salud de las Comunidades Autónomas y éstos al Instituto Nacional de Seguridad Social, el cual una vez realizadas las compensaciones por los gastos ocasionados por españoles en otros Estados Miembros, gestiona los pagos correspondientes a cada una de las Comunidades Autónomas 32.

Los pacientes comunitarios del Sistema Nacional de Salud español se pueden agrupar en tres grupos: (1) visitantes de corta duración (turistas, ejecutivos o estudiantes), (2) residentes (jubilados) y (3) personas que viven en las fronteras o cerca de estas 32,33. Los servicios de urgencia son los más solicitados por los turistas, mientras que los residentes presentan demandas semejantes a los nacionales. Los residentes que tienen España como segunda residencia pueden bien utilizar la Tarjeta Sanitaria Europea para cortas estancias, o empadronarse como residentes y utilizar la Tarjeta Individual Sanitaria del Sistema Nacional de Salud. No obstante, algunos residentes, al no empadronarse y regularizar su situación sanitaria forman una población flotante difícil de conocer.

Por otro lado, en los territorios fronterizos de España con Francia y Portugal existen diversos acuerdos sanitarios. Así, en la frontera franco-española se ha creado un hospital que sirve poblaciones de ambos lados, Hospital Transfronterizo de la Cerdanya, y en la frontera hispano-portuguesa la Comunidad Autónoma de Extremadura y las autoridades sanitarias de Portugal tienen un Acuerdo para atender en España a ciudadanos de ciudades portuguesas fronterizas. Ambos programas cuentan con el apoyo de fondos europeos.

\section{Consideraciones sobre el proceso de integración europea y el sector salud: lecciones de la experiencia española.}

La UE tiene un impacto real en la vida de sus ciudadanos. La pertenencia a la UE ha supuesto una auténtica revolución para decenas de millones de europeos, especialmente los del Oeste (España y Portugal) y Este de Europa, países que recibieron un nuevo impulso a sus democracias recientes, que vieron caer fronteras de siglos por las que ahora circulan libremente bienes y personas, haciendo posible, entre otras cosas, que cientos 
de miles de jóvenes pueden estudiar con el Programa Erasmus en cualquier país de la Unión. La ubicuidad de la legislación de origen comunitario en la vida diaria y el euro como moneda común son dos de los signos más visibles de la UE en la vida de todos los europeos.

Sin duda alguna, la UE está viviendo un periodo de incertidumbres tras el estancamiento de la Constitución Europea, las discrepancias internas sobre las fronteras y el futuro de la Unión, así como su papel en el mundo actual. Ante ello es bueno recordar la famosa frase de Jacques Delors: Europa se construye a golpe de crisis. En este sentido la Unión se enfrenta a retos importantes y difíciles, pero la UE misma no está en cuestión como modelo de integración que se sostiene en una comunidad de intereses y valores compartidos, entre los que destacan, derechos humanos, libertad, igualdad, democracia, justicia social.

Uno de los desafíos más importantes de la UE es seguir avanzando en competitividad y crecimiento económico, asumir el reto de protección del medio ambiente, al tiempo que incrementa la protección y cohesión social dentro de cada Estado y en el conjunto de la Unión - que cuenta para ello con un importante presupuesto. El modelo social europeo puede ser considerado como el denominador común de los valores y características compartidos por el conjunto de los Estados de bienestar de cada uno de los Estados Miembros de la UE. Es una de las señas de identidad de la Unión, pero también un punto de tensión entre los que desean una Europa más social y política, y los que ponen el acento en la competitividad y crecimiento económico, enfatizando más el objetivo de un mercado sin fronteras que un proyecto de integración político y social. El rechazo a la Constitución Europea en Francia y Holanda, es todo un signo de distanciamiento entre algunos ciudadanos y el proyecto europeo, así como de las dificultades y retos de la integración europea.

El proceso de integración europeo ha sido muy gradual y en gran medida se ha ido haciendo camino al andar. En este proceso, los temas de salud no han sido nunca centrales ni prioritarios; se han ido incorporando a los tratados a medida que avanzaba el mercado interior, cada vez con más peso en la agenda comunitaria. Como era de esperar, los temas de salud tienden ser más importantes según avanza el proceso de integración y la movilidad dentro de la EU. Por otro lado, en los últimos años, especialmente a partir de la crisis de las vacas locas, las crisis internacionales de salud pública (amenaza de la gripe aviar) han contribuido a reforzar en la UE la construcción de un sistema de Salud Pública, respaldado por agencias específicas.
A pesar del principio de subsidiariedad, los temas de salud ganan relevancia en la agenda europea, de modo que no sólo la protección de la salud, sino también su promoción están entrando de lleno en las actividades de la comisión y del parlamento. Por su parte, la Agenda de Lisboa reconoce el papel de la buena salud como factor de desarrollo económico y el sector salud adquiere relevancia como de los sectores más dinámicos de la Unión, no sólo por su contribución a la salud y bienestar colectivos, sino por el empleo que genera, su papel en la innovación y desarrollo tecnológicos. Por todo ello, la investigación biomédica básica y aplicada, la industria farmacéutica y de aparatos médicos, las tecnologías de la información vinculadas a la sanidad constituyen líneas de actividad prioritaria de la UE.

Es importante destacar en las conclusiones de esta revisión que los temas de salud pública, vinculados directa o indirectamente a la protección de la salud y la seguridad de los consumidores, son el aspecto más relevante relacionado con la salud en la UE. Ello es en primer lugar una consecuencia directa en un contexto democrático de la lógica del mercado común interior y de las reglas de la competencia, pero también de la voluntad política de garantizar a todos los ciudadanos de la Unión por igual un alto nivel de protección de la salud.

Esta es quizá la lección más importante en relación a la salud para otros procesos de integración regional: es obligado que los ciudadanos de un espacio económico común tengan iguales garantías de seguridad y protección a la salud respecto a los productos (industriales, alimenticios, medicamentos, juguetes) en circulación en el mismo, que existan estándares comunes obligatorios de seguridad e higiene en el trabajo, así como en relación al medio ambiente.

Por todo ello, lo fundamental en la construcción de una Europa de la salud ha sido la puesta en marcha de un sistema de salud pública, de garantías de protección a la salud y seguridad para los consumidores y usuarios de todo tipo productos en el mercado interior común. Este ha sido uno de los principales esfuerzos regulatorios de la UE, e incluye la protección del medio ambiente y de la seguridad e higiene en el trabajo, que es imprescindible para que la competencia y libre mercado interior estén basados en calidad, innovación y precio, no en bajos niveles de higiene y seguridad de los trabajadores o en la degradación medioambiental. Establecer los correspondientes estándares, evaluar viejos y nuevos riesgos, regular todos aquellos sectores que pueden afectar a la seguridad y salud y vigilar la implementación de las normas es una tarea permanente de la Comisión. En los últimos años, 
esta dinámica ha llevado al análisis sistemático de las implicaciones para la salud de todas las políticas de la Unión. En este sentido el proceso de integración - que no es únicamente la adhesión de un Estado a la Unión, sino la evolución de la propia Unión - actúa como un motor de desarrollo de políticas y estándares protección a la salud y al medio ambiente.

Desde este punto de vista, una parte importante del impacto de la UE en los servicios sanitarios de los Estados Miembros a través de la regulación del mercado interior puede ser visto como una extensión de las políticas de protección de la salud y seguridad de los consumidores. No es otro el objetivo último de la regulación de medicamentos y aparatos médicos o de la homologación de las titulaciones profesionales del personal sanitario, cuyo impacto hemos visto en el caso español.

En España, el efecto positivo de la regulación europea se ha notado especialmente en la mejor de la protección de la salud: higiene y seguridad alimentaria, medio ambiente, seguridad e higiene en el trabajo, normas de seguridad de equipos y bienes de consumo. Como se ha señalado, en los servicios sanitarios la normativa europea impregna toda la legislación sobre medicamentos, aparatos médicos, formación de profesionales y contratación de bienes y servicios. Desde otra perspectiva más indirecta - pero no poco importante - la sanidad pública española y los indicadores de salud de la población, se han beneficiado los efectos positivos de la integración en la UE derivados del crecimiento económico sostenido, de la estabilidad macroeconómica, de la cohesión social y de la consolidación de la institucionalización política.

En el ámbito interno, es interesante señalar que siendo la salud pública constitucionalmente competencia de las Comunidades Autónomas y gestionando éstas los servicios sanitarios, el Gobierno central se encuentra en un papel de intermediario entre las instituciones de la UE y los gobiernos de las Comunidades Autónomas. De ahí la demanda de que las Comunidades Au- tónomas participen en la formación de la voluntad de España en la UE en estas materias que les afectan. Para ello se han encontrado fórmulas de presencia y participación de las Comunidades Autónomas en las delegaciones españolas ante la UE, muy similares a las existentes en Alemania. Esta problemática podría ser muy similar en los Estados federales del MERCOSUR.

En relación con la conformación de la voluntad europea manifestada en las directivas es importante señalar que éstas son el resultado de un complejo proceso técnico-político al que los Estados miembros contribuyen decisivamente a través de las Representaciones Permanentes ante la Comisión, en el Consejo y en diversos Comités. En este proceso parten con ventaja para defender sus posiciones e intereses aquellos Estados Miembros que cuentan con la ventaja competitiva de una administración profesionalizada y estable. Este es un aspecto en el que existen grandes diferencias entre los Estados Miembros de la UE, donde los altos cargos de los países del sur cambian con el ciclo político, en contraste con los del norte. Este hecho coloca a aquellos en desventaja en las negociaciones internas dentro de la UE. Esta desventaja comparativa deriva tanto de los funcionarios que periódicamente llegan nuevos a un mundo que desconocen, como de la ineficiencia de una administración pública no profesionalizada, basada en la ocupación político-partidaria de sus altos cargos. Esta es una lección de gran importancia, tanto por su impacto en los escenarios supra estatales como, sobre todo, por las consecuencias negativas administraciones públicas alejadas de las prácticas del buen gobierno.

Por último, hemos visto que proceso de integración desde la UE y desde España no ha sido, y no es, un proyecto sin problemas o con problemas menores. Sin embargo, no cabe la menor duda que la economía, la cohesión y protección social, la democracia, la salud pública y los servicios sanitarios que tendrían dificultades mucho mayores y mucho menos susceptibles de solución si la UE no existiera. 


\section{Resumen}

El texto revisa el impacto de la integración europea en el sector salud (salud pública y servicios sanitarios), estudiando instituciones, funcionamiento y competencias de la Unión Europea (UE), a partir de literatura, documentos y observaciones coyunturales. La UE no tiene competencias directas en salud, pero su legislación ejerce importante impacto en las políticas sanitarias de todos los Estados Miembros. Esta influencia se manifiesta en la protección de la salud y seguridad de los consumidores, regulación de medicamentos y aparatos médicos, homologación de títulos profesionales, libertad de movimiento de profesionales y pacientes, compras y contratación, e investigación biomédica. Estudiando la evolución de la política de salud en la UE se constata un refuerzo progresivo de sus políticas sanitarias y que el objetivo de alcanzar un alto nivel de protección de la salud, tiene importantes implicaciones en todas las políticas europeas. Tomando como caso de estudio las repercusiones de la integración europea en el sistema sanitario español, se destacan sus aspectos más influyentes, retos actuales y perspectivas, así como lecciones para que procesos de integración regional sean factores de equidad y eficiencia en salud.

Unión Europea; Política de Salud; Sistemas de Salud

\section{Referencias}

1. Guimarães L, Giovanella L. Integração européia e políticas de saúde: repercussões do mercado interior europeu no acesso aos serviços de saúde. Cad Saúde Pública 2006 22:1795-807.

2. Comisión Europea. Eurostat: Population et conditions sociales. http://www.eu.int/comm/eurostat (accedido el 24/May/2006).

3. Barón-Crespo E. Europa en el alba de milenio. Madrid: Acento Editorial; 1994

4. Comisión Europea. El abc del derecho comunitario. Luxemburgo: Oficina de Publicaciones Oficiales de las Comunidades Europeas; 2000. (Serie Documentación Europea).

5. Comisión Europea. Instituciones y otros órganos de la Unión Europea. Instituciones de la UE. http://europa.eu/institutions/inst/index_es.htm (accedido el 19/Jul/2006).

6. Comisión Europea. El funcionamiento de la Unión Europea. Guía del ciudadano sobre las instituciones de la Unión Europea. Luxemburgo: Oficina de Publicaciones Oficiales de las Comunidades Europeas; 2003. (Serie Documentación Europea).

7. Mansito F. Sobre la integración europea. Se hace camino al andar. Revista Información Comercial Española 2006; (831):17-29.

\section{Colaboradores}

L. Guimarães y J.-M. Freire comparten la autoría del artículo en la concepción, estructura, búsqueda de fuentes de referencias y análisis para la construcción del texto, y en la aprobación final para la publicación. L. Guimarães ha preparado una primera versión del texto, posteriormente revisada y elaborada conjuntamente por los dos autores.

\section{Agradecimientos}

El artículo ha sido producido durante el Programa de Pasantía en el Exterior, de la Coordinación de Perfeccionamiento de Personal de Nivel Superior, realizado en la Escuela Nacional de Sanidad, del Instituto de Salud Carlos III, España. L. Guimarães es becaria del Conselho Nacional de Desenvolvimento Científico e Tecnológico y de la Coordenação de Aperfeiçoamento de Pessoal de Nível Superior y funcionaria del Ministério da Saúde de Brasil.
8. Comisión Europea. Al servicio del ciudadano europeo. Como trabaja la Comisión Europea Luxemburgo: Oficina de Publicaciones Oficiales de las Comunidades Europeas; 2003. (Serie Europa en Movimiento)

9. Comisión Europea. Los procedimientos decisorios de la Unión. El principio de subsidiariedad y el cometido de los parlamentos nacionales. http:// europa.eu/scadplus/constitution/subsidiarity es.htm (accedido el 16/Jul/2006).

10. Comisión Europea. Doce lecciones sobre Europa Luxemburgo: Oficina de Publicaciones Oficiales de las Comunidades Europeas; 2003. (Serie Documentación Europea).

11. Comunidades Europeas. Una constitución para Europa - constitución adoptada por jefes de Estado o de Gobierno. Presentación a los ciudadanos. Luxemburgo: Oficina de Publicaciones Oficiales de las Comunidades Europeas; 2004.

12. Paton C, Berman PC, Busse R, Ong BN, Rehnberg C, Renck B, et al. The Europe union and health services: summary. In: Busse R, Wismar M, Berman PC, editors. The European Union and health services. The impact of the single European market on member states. Amsterdam: IOS Press; 2002. p. 1-13. 
13. Silió-Villamil F, Romo-Avilés N, Prieto-Rodríguez MA. The SEM and the public procurement of goods and services in the Andalusian health service. In: Busse R, Wismar M, Berman PC, editors. The European Union and health services. The impact of the single European market on member States. Amsterdam: IOS Press; 2002. p. 179-211.

14. Koivusalo MT. The future of European health policies. Int J Health Serv 2005; 35:325-42.

15. Comisión Europea. Tratado de la Unión Europea. Diario Oficial no. C191 de 29 de julio de 1992. http://europa.eu.int/eur-lex/lex/es/treaties/ dat/11992M/htm/11992M.html (accedido el 16/ $\mathrm{Jul} / 2006)$.

16. Comisión Europea. Tratado de Ámsterdam por el que se modifican el Tratado de la Unión Europea, los Tratados Constitutivos de las Comunidades Europeas y determinados actos conexos. Diario Oficial $\mathrm{n}^{\circ}$. C340 de 10 de noviembre de 1997. http://europa.eu.int/eur-lex/lex/es/treaties/dat/ 11997E/htm/11997E.html\#0173010078 (accedido el 16/Jul/2006).

17. European Commission, Directorate-General Health and Consumer Protection. The contribution of health to the economy in the European Union. Luxembourg: Office for Official Publications of the European Communities; 2005.

18. Wismar M, Lahtinen E, Stahl T, Ollila E, Kimmo L. Introduction. In: Stahl T, Wismar M, Ollila E, Lahtinen E, Leppo K, editors. Health in all policies. Prospects and potentials. Finland: Finnish Ministry of Social Affairs and Health; 2006. p. xvii-xxx.

19. Comisión Europea. DG Sanidad y protección de los consumidores. Salud pública. http://ec.europa.eu/ health/index_es.htm (accedido el 31/May/2006).

20. Comisión Europea. Perspectiva de la política sanitaria. http://ec.europa.eu/comm/health/index_ es.htm (accedido el 31/May/2006).

21. Villamil FS, Rodríguez MAP, Avilés NR. Impacto de las Políticas de la Unión Europea sobre los Sistemas Sanitarios. In: Cabasés JM, Villalbí JR, Airbar C, editores. Invertir para la Salud. Prioridades en salud pública. v. II. Barcelona: Sociedad Española de Salud Pública y Administración Sanitaria; 2002. p. 343-76. (Informe de la Sociedad Española de Salud Pública y Administración Sanitaria).

22. Noack RH. Whiter? Public health in a changing Europe [Editorial]. Eur J Public Health 2005; 15:442.

23. Condé EAS. Laços da diversidade: a Europa social e o welfare em movimento (1992-2002) [Tesis de Doctorado]. São Paulo: Instituto de Economia,Universidade Estadual de Campinas; 2004.

24. Cabiedes L, Guillén A. ¿̇Hacia la convergencia de los sistemas de asistencia sanitaria europeos? In: Moreno L, editor. Unión Europea y Estado del Bienestar. Madrid: Consejo Superior de Investigaciones Científicas, Instituto de Estudios Sociales Avanzados; 1997. p. 373-403.

25. McKee M. European health policy: where now? [Editorial]. Eur J Public Health 2005; 15:557-8.
26. Wismar M, Busse R, Paton C, Silió-Villamil F, Romo-Avilés N, Prieto-Rodríguez MA, et al. Transposition of European directives into national legislation. In: Busse R, Wismar M, Berman PC, editores. The European Union and health services. The impact of the single European market on member states. Amsterdam: IOS Press; 2002. p. 49-59.

27. Abel-Smith B, Figueras J, Holland W, McKee M, Mossialos E. Choices in health policy - an agenda for the European Union. Luxembourg: Dartmounth Publishing Company Limited; 1995.

28. Worz M, Foubister T, Busse R. Access to health care in the EU member states. Euro Observer 2006; 8: $1-4$.

29. Hubel M, Price C. Action by the European Union on health inequalities. Eurohealth 2005; 11:4-5.

30. Freire JM. El Sistema sanitario público: perspectivas de futuro. In: Fernández-García T, Marin Sánchez M, editores. Estado de bienestar y socialdemocracia: ideas para el debate. Madrid: Alianza Editorial; 2001. p. 129-78.

31. Iñesta García A. Políticas de medicamentos. In: Repullo JR, Ochoa LAO, editores. Un nuevo contrato social para un Sistema Nacional de Salud sostenible. Barcelona: Editora Ariel SA; 2005. p. 131-61.

32. Rosenmoller M, McKee M, Baeten R, Glinos IA. Patient mobility: context and issues. In: Rosenmoller M, McKee M, Baeten R, editors. Patient mobility in the European Union-learning from experience. Copenhagem: The European Observatory on Health Systems and Policies/World Health Organization; 2006. p. 1-7.

33. Rosenmoller M, Lluch M. Meeting the needs of long-term residents in Spain. In: Rosenmoller M, McKee M, Baeten R, editors. Patient mobility in the European Union -learning from experience. Copenhagen: The European Observatory on Health Systems and Policies/World Health Organization; 2006. p. 59-78.

34. Gobrecht J, Busse R. Consumer Choice of Medical Goods across Borders. In: Busse R, Wismar M, Berman PC, editors. The European Union and health services. The impact of the single European market on member states. Amsterdam: IOS Press; 2002. p. 213-30.

35. Comisión Europea. Consulta sobre la acción comunitaria en materia de servicios de salud. http:// ec.europa.eu/health/ph_overview/co_operation/ mobility/patient_mobility_es.htm (accedido el 08/Feb/2007).

36. Wismar M, Busse R, Berman P. The Europe Union and health services - the context. In: Busse R, Wismar M, Berman PC, editors. The European Union and health services. The impact of the single European market on member states. Amsterdam: IOS Press; 2002. p. 17-29.

37. Cortes Españolas. Constitución Española de 27 de diciembre de 1978. Boletín Oficial del Estado; 29 dic. http://www.boe.es/g/es/bases_datos/doc. php?coleccion=iberlex\&id=1986/10499 (accedido el 08/Feb/2007). 
38. Stuart AM. Regionalismo e democracia: o surgimento da dimensão subnacional na União Européia. In: Vigevani T, Wanderley LE, Barreto MI, Mariano MP, editores. A dimensão subnacional e as relações internacionais. São Paulo: EDUSC; 2004. p. 109-57.

39. Medeiros MA. Unidades subnacionais e integração européia: o caso do comitê das regiões.In: Vigevani T, Wanderley LE, Barreto MI, Mariano MP, editores. A dimensão subnacional e as relações internacionais. São Paulo: EDUSC; 2004. p. 159-77.

40. Ley General de Sanidad. Ley 14/1986, de 25 de abril, Boletín Oficial del Estado no. 102; 29 abr. http://www.congreso.es/constitucion/ficheros / leyes_espa/1_014_1986.pdf (accedido el 16/Sep/ 2006).

41. Ministerio de Sanidad y Consumo. Sistema nacional de salud. Madrid: Artes Gráficas Palermo; 2006.

42. Freire JM. La cobertura poblacional del Sistema Nacional de Sanidad: importancia y retos de la universalización y equidad en el aseguramiento. In: Repullo-Labrador JR, Oteo-Ochoa LA, editores. Un nuevo contrato social para un Sistema Nacional de Salud sostenible. Barcelona: Editora Ariel; 2005. p. 61-99.
43. Freire JM. La atención primaria de la salud y los hospitales en el Sistema Nacional de Salud. In: Navarro-Lopez V, editor. El Estado de bienestar en España. Madrid: Tecnos; 2004. p. 239-91.

44. Guillén A, Cabiedes L. Towards a National Health Service in Spain: the search for equity and efficiency. J Eur Social Policy 1997; 7:319-36.

45. Cabiedes L. Reformas recientes en el sector del medicamento en España. In: Terceras Jornadas Santafesinas y Segundas Federales Política, Economía y Gestión de Medicamentos. Santa Fe: Colegio de Farmacéuticos de la Provincia de Santa Fe - 1a Circunscripción; 2004. p. 169-95.

46. Otero O, Martín-Moreno JM, Castellón E. El sistema sanitario español y las reformas en Europa. In: Saltman RB, Figueras J, editor. Reformas sanitarias en Europa: análisis de las estrategias actuales. Madrid: Secretaría General Técnica, Ministerio de Sanidad y Consumo; 1997. p. 343-66.

Recibido el 20/Oct/2006

Versión final presentada el 12/Feb/2007

Aprobado el 02/Mar/2007 